\title{
The Improvement of Circuit Power Factor
}

\author{
Xuan Wu \\ School of North China Electric Power University, Baoding 071000, China. \\ 3336180262@qq.com
}

\begin{abstract}
In the power system, the power factor of the grid is low due to the influence of load and transmission equipment. In the face of a large number of reactive loads in the power grid, compensating reactive power and suppressing harmonic current can effectively improve the power factor of the power grid, reduce damage to electrical equipment, and improve power supply efficiency. This paper first introduces the reactive power compensation in detail, and combines with the fluorescent lamp circuit for analysis. Then combined with the recent harmonic suppression method to analyze the advantages and disadvantages, the role and principle of harmonic suppression are explained in detail.
\end{abstract}

Keywords: Power factor, inductive load, reactive power compensation, harmonic suppression.

\section{Introduction}

Most loads in power systems are inductive loads such as transformers, motors, fluorescent lamps, and so on. It is characterized by the current of the load lags behind the voltage by one phase. Simultaneous operation of multiple inductive loads in the circuit is detrimental to the power system, making the capacity of the power supply insufficiently utilized. And because the power factor of the inductive load is low, the entire circuit needs to provide a higher power factor to meet the load requirements. Under the premise that the given voltage is constant, if the current value is increased, a large power transmission loss power will be caused, so that the transmission efficiency is greatly reduced.

In order to satisfy as high a power factor as possible during load operation, reactive power compensation and harmonic suppression are usually used.

\section{Reactive Power}

The generator is connected to the constant voltage AC grid, so the total flux value (including field flux, armature reaction flux and armature leakage flux) of the armature winding crosslink should remain unchanged regardless of the excitation variation. When overexcited, the excitation flux increases. In order to keep the total magnetic flux of the armature unchanged, the demagnetization component of the armature reaction will increase, and the angle between I and E0 will increase, so the current lags behind the voltage. Then the generator emits inductive reactive power.

It can be seen that when the prime mover power is constant, changing the previous current will cause a change in the reactive power of the motor, and the total stator current will change. When the excitation current is equal to the normal excitation value, the armature current value is the smallest, and on this basis, the stator current will increase regardless of the increase or decrease of the excitation current.

\section{Reactive Power Compensation}

\subsection{Classification of Shunt Capacitor Compensation.}

In many engineering power supply systems, shunt capacitors are often used to compensate reactive power and improve power factor.

Depending on the installation location, shunt capacitor compensation can be divided into three categories depending on the installation location. The first type is to install the capacitor bank on the busbar in order to improve the power factor of the entire substation and reduce the reactive loss of 
the feeder circuit. The second type is partition compensation. The capacitor banks are respectively installed on the regional bus with lower power factor. The compensation effect of the partition compensation is better, but the compensation range is smaller than that of the centralized compensation. The third type is local compensation. For inductive devices such as asynchronous motors and fluorescent lamps, the capacitor bank is installed near the load device to perform reactive compensation on the spot. The advantage of this method is that it can't only improve the power factor of the power supply circuit of the power equipment, but also improve the voltage quality of the power equipment, and has a wide range of applications in China.

\subsection{Compensation of Fundamental Reactive Power in Fluorescent Lamp Circuit.}

In many engineering power supply systems, shunt capacitors are often used to compensate reactive power and improve power factor.

Depending on the installation location, shunt capacitor compensation can be divided into three categories depending on the installation location. The first type is to install the capacitor bank on the busbar in order to improve the power factor of the entire substation and reduce the reactive loss of the feeder circuit. The second type is partition compensation. The capacitor banks are respectively installed on the regional bus with lower power factor. The compensation effect of the partition compensation is better, but the compensation range is smaller than that of the centralized compensation. The third type is local compensation. For inductive devices such as asynchronous motors and fluorescent lamps, the capacitor bank is installed near the load device to perform reactive compensation on the spot. The advantage of this method is that it can't only improve the power factor of the power supply circuit of the power equipment, but also improve the voltage quality of the power equipment, and has a wide range of applications in China.

\subsubsection{Principle of Fluorescent Lamps}

There is a small filament electrode at each end of the fluorescent tube. The glass tube is coated with a fluorescent substance, and the tube is evacuated and then filled with a small amount of inert gas and a small amount of mercury. When a high voltage is applied between the filaments at both ends, an arc is released in the tube, and the mercury vapor is excited to radiate plenty of ultraviolet rays, and the phosphor on the tube wall emits visible light under the excitation of ultraviolet rays.

When the fluorescent lamp circuit turns on the switch to raise the voltage, the voltage is applied to the ballast, the filament resistor and the starter, and the voltage across the lamp is not enough to cause the arc to be placed, and the glow discharge is generated between the two stages of the starter. Then the moving piece is heated and deformed to contact with the fixed piece, the glow discharge stops, the moving piece cools and shrinks and resets, and the connected circuit is disconnected. At this time, due to the sudden disconnection of the circuit, the ballast (inductor with iron core) is higher. The selfinductance voltage, high voltage load on both ends of the lamp tube, causes the arc to be placed in the tube, and the phosphor is excited to emit visible light. When the fluorescent lamp is working normally, the voltage across the lamp is lower than the operating voltage of the starter, and the starter is no longer active.

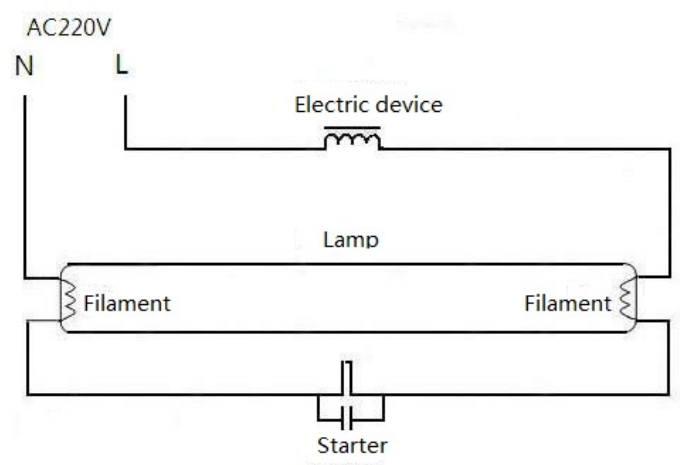

Fig. 1 Fluorescent lamp schematic 


\subsubsection{Circuit Principle}

In fact, the fluorescent lamp circuit is equivalent to a series circuit of a resistor and an inductor.

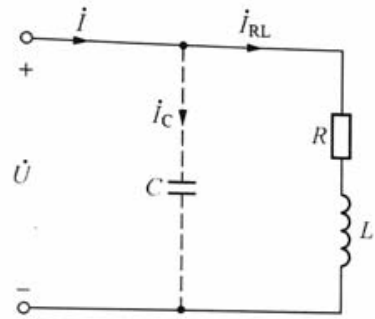

(a)



(b)

Fig. 2 Circuit diagram

\section{Harmonic Suppression Method}

\subsection{Harmonic}

The harmonic is a sine wave component that is segmented from the periodic electrical quantity. From the frequency value, the harmonic is usually an integer multiple of the fundamental wave. Fourier series decomposition is performed on the periodic non-sinusoidal electric quantity, wherein the electric current generated by the current larger than the fundamental frequency is the harmonic we are concerned about.

In power systems, the presence of higher harmonics can cause grid voltage fluctuations and threephase imbalances, affecting power quality, transmission efficiency, and safe operation of equipment. The power factor of the circuit can be increased by suppressing harmonics.

Normally, the power industry uses a sinusoidal waveform distortion rate when verifying the system's current distortion.

$$
T H D_{i}=\sqrt{\sum_{n=2}^{\infty} I_{n}^{2}} / I_{1} \times 100 \%
$$

I1: the rated fundamental current

In: the effective value of the harmonic current.

\subsection{Method of Suppressing Harmonics}

In order to prevent harmonics from flowing into the public power grid and equipment in the power grid system, an external filtering and reactive power compensation device can be used. The main methods are to add a passive filter (PPF), add an active filter (APF), and add a hybrid active filter (HAPF).

The methods currently used in engineering are passive LC filters and active power filters.

The principle of passive filter filtering harmonics is essentially to provide a low-impedance path for harmonics in the circuit, so that harmonics can pass through the filter without injecting into the system that is, leaving the fundamental wave and short-circuiting the harmonics. The PPF can also be designed as a high-pass filter to filter out certain higher harmonics in a targeted manner.

The principle of the active filter is to use a controllable power semiconductor device to inject a current equal to the amplitude of the harmonic source current and the opposite phase to the grid, so that the total harmonic current of the power supply is zero, and the real-time compensation of the harmonic current is achieved. The harmonic source is generally a non-linear load, generating a harmonic current (Ih). The active power filter is equivalent to a flow control current source, and its function is to generate a compensation current having the same amplitude and opposite phase as the harmonic source harmonic current (Ih) to achieve the purpose of eliminating harmonics.

If you follow the "checklist" your paper will conform to the requirements of the publisher and facilitate a problem-free publication process. 


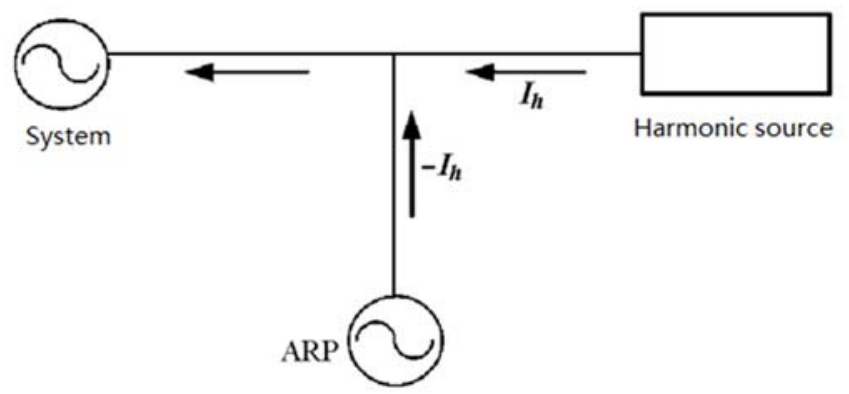

Fig. 3 Active power filter schematic

\section{References}

[1]. Kang, M. \& Zhiqiang. W., et al. Improvement of power factor of non-sinusoidal circuits. [J]. Journal of Shaanxi University of Technology. Vol. 23 (2007) No. 03, p. 20-25.

[2]. Xiuchen Jia: Application of Hybrid Active Power Filter in Harmonic Suppression and Reactive Power Compensation in Casting and Forging Enterprises. (D, Jiangsu University, China 2017). P.1-58. 